\title{
DIMENSIONS AND MEASURES OF QUASI SELF-SIMILAR SETS
}

\author{
K. J. FALCONER
}

(Communicated by Kenneth R. Meyer)

\begin{abstract}
We show that sets with certain quasi self-similar properties have equal Hausdorff and box-packing dimensions and also have positive and finite Hausdorff measure at the dimensional value. A number of applications of these results to particular examples are given.
\end{abstract}

\section{INTRODUCTION AND DEFINITIONS}

The idea of dimension is fundamental in the study of fractals, see Mandelbrot [5]. Various definitions of dimension have been proposed, such as the Hausdorff dimension, which is mathematically convenient being based on Hausdorff measures, and the box counting dimensions, which are computationally convenient. It is often desirable to know whether such differing definitions give the same dimensional value for a particular fractal. Moreover, it is very useful to know whether a set of Hausdorff dimension $s$ is an $s$-set, that is whether the $s$-dimensional Hausdorff measure is positive and finite. Much of the fundamental work done by Besicovitch and others (see Falconer [3]) relates specifically to sets that satisfy this rather strong condition.

Rigorous calculations of Hausdorff dimension normally involve calculating the $s$-dimensional Hausdorff measures of a set, and then finding the value of $s$ at which the measure jumps from infinity to zero. Calculation of such measures often requires careful estimates involving admissible coverings of the set. Similarly, careful estimates are required to find box counting dimensions before these dimensional values may be compared.

This paper discusses the opposite approach. We give conditions on a set of Hausdorff dimension $s$ that guarantee that the $s$-dimensional Hausdorff measure is positive and/or finite without any need to calculate the actual value of $s$. From this information, $s$ can often be found easily. (For example, given that the middle third Cantor set has positive finite measure at the critical value, it is a trivial exercise using the scaling property of Hausdorff measures to show that the Hausdorff dimension is $\log 2 / \log 3$.) Similarly, we give conditions that

Received by the editors May 10, 1988 and, in revised form September 5, 1988.

1980 Mathematics Subject Classification (1985 Revision). Primary 58F12, 28 A75.

Key words and phrases. Dimension, fractal, self-similar set, repeller. 
ensure that the box-counting and Hausdorff dimensions are equal without the need to evaluate them.

We call these theorems implicit in that they enable us to draw conclusions about dimensions that are not explicitly calculated. The first result of this nature, which we quote as Theorem 1, was due to McLaughlin [6]. Our results are applicable to fractals that are renormalisable in some sense or that have a quasi self-similar structure. We give a number of such applications in $\S 3$.

Let $(F, d)$ be a metric space. For $0 \leq s<\infty$ the $s$-dimensional Hausdorff outer measure of $F$ is given in the usual way by

$$
\mathscr{H}^{s}(F)=\lim _{\delta \rightarrow 0} \inf \left\{\sum_{1}\left|U_{i}\right|^{s}: F \subseteq \bigcup_{1} U_{i} ; 0<\left|U_{1}\right|<\delta\right\},
$$

where || denotes the diameter of a set. This leads to the definition of the Hausdorff dimension of $F$ :

$$
\operatorname{dim}_{H} F=\inf \left\{s: \mathscr{H}^{s}(F)<\infty\right\}=\sup \left\{s: \mathscr{H}^{s}(F)>0\right\} .
$$

Although the usual situation is of a fractal $F$ embedded in some larger metric space, usually $\mathbf{R}^{n}$, for many purposes, such as in the above definitions, we may work entirely with the restriction of the metric to $F$.

Many other definitions of dimension are encountered in the literature, some more satisfactory and useful than others. Let $(F, d)$ be a metric space, and, for $\varepsilon>0$, let $n(\varepsilon)$ be the smallest number of sets of diameter at most $\varepsilon$ that cover $F$. We define the lower and upper box counting dimensions by

$$
\begin{aligned}
& \underline{\operatorname{dim}}_{B} F=\underline{\lim }-\log n(\varepsilon) / \log \varepsilon \\
& \overline{\operatorname{dim}}_{B} F=\varlimsup_{\varepsilon \rightarrow 0}-\log n(\varepsilon) / \log \varepsilon .
\end{aligned}
$$

We get exactly the same values if we take $n(\varepsilon)$ to be the smallest number of closed balls of radii $\varepsilon$ with centres in $F$ that cover $F$. Moreover, by relating $n(\varepsilon)$ and $n(2 \varepsilon)$, a simple exercise shows that we obtain the same values for ${\operatorname{dim}_{B}}_{B}$ and $\overline{\operatorname{dim}}_{B}$ if, in (1.1) and (1.2), we let $n(\varepsilon)$ be the largest number of disjoint closed balls all of radii $\varepsilon$ with centres in $F$. There are other equivalent formulations of the box counting dimensions; different ones are convenient in different situations.

The important difference between Hausdorff dimensions and the box counting dimensions is that in calculating Hausdorff dimensions different weights $|U|^{s}$ are attached to covering sets $U$, whereas in caculating box counting dimensions, the same weight $\varepsilon^{s}$ is attached to each covering set (notice that $\underline{\operatorname{dim}}_{B} F=\inf \left\{s: \underline{\lim } n(\varepsilon) \varepsilon^{s}<\infty\right\}$, etc.). From the definitions,

$$
\operatorname{dim}_{H} F \leq \underline{\operatorname{dim}}_{B} F \leq \overline{\operatorname{dim}}_{B} F .
$$

In a certain sense, the Hausdorff dimension and upper box counting dimension may be regarded as the smallest and greatest values of any reasonable definition 
of dimension. For example, the packing dimension of Tricot [7] always lies between these two values.

\section{IMPLICIT THEOREMS}

For completeness we quote the result of McLaughlin [6], and then give a minor, but useful, generalisation.

Theorem 1. Let $(F, d)$ be a nonempty compact metric space, with $\operatorname{dim}_{H} F=s$. Suppose that there exist $\lambda_{0}, a>0$ such that for any set $N \subseteq F$ with $|N|<\lambda_{0}$ there is a mapping $\varphi: N \rightarrow F$ such that

$$
\operatorname{ad}(x, y) \leq|N| d(\varphi(x), \varphi(y))
$$

for $x, y \in N$. Then $a^{s} \leq \mathscr{M}^{s}(F)$.

Proof. This is the result of McLaughlin [6] with only minor modification.

Theorem 2. Let $(F, d)$ be a nonempty compact metric space, with $\operatorname{dim}_{H} F=s$. Suppose that there exist $\lambda_{0}, a>0$ and an integer $m$ such that for any set $N \subseteq F$ with $|N|<\lambda_{0}$ there are sets $N_{j}$ with $N \subseteq \bigcup_{j=1}^{m} N_{j}$ and mappings $\varphi_{j}: N_{j} \rightarrow F(1 \leq j \leq m)$ such that

$$
\operatorname{ad}(x, y) \leq|N| d\left(\varphi_{j}(x), \varphi_{j}(y)\right)
$$

for $x, y \in N_{j}$. Then $a^{s} m^{-1} \leq \mathscr{H}^{s}(F)$.

Proof. Suppose that $0 \leq \mathscr{H}^{s}(F)<a^{s} m^{-1}$. Then given $0<\delta<\min \left\{\lambda_{0}, \frac{1}{2} a\right\}$ we may find sets $U_{1}, \ldots, U_{k}$ with $\left|U_{1}\right|<\delta$ and $F \subseteq \bigcup_{i=1}^{k} U_{1}$ such that

$$
\sum_{i=1}^{k}\left|U_{i}\right|^{s}<a^{s} m^{-1}
$$

and so with

$$
\sum_{i=1}^{k}\left|U_{i}\right|^{t}<a^{t} m^{-1}
$$

for some $t<s$. By the hypotheses of the theorem we may find sets $U_{i, j}$ and mappings $\varphi_{i, j}: U_{i, j} \rightarrow F \quad(1 \leq j \leq m)$ such that $U_{i} \subset \bigcup_{j=1}^{m} U_{i, j}$ and

$$
\operatorname{ad}(x, y)<\left|U_{i}\right| d\left(\varphi_{i, j}(x), \varphi_{i, j}(y)\right)
$$

for $x, y \in U_{i, j}$. Thus we have $\left|\varphi_{i, j}^{-1}\left(U_{q}\right)\right|<a^{-1}\left|U_{i}\right|\left|U_{q}\right|<\frac{1}{2} \delta$ and

$$
\sum_{i=1}^{k} \sum_{j=1}^{m} \sum_{q=1}^{k}\left|\varphi_{i, j}^{-1}\left(U_{q}\right)\right|^{t}<m a^{-t}\left(\sum_{i=1}^{k}\left|U_{i}\right|^{t}\right)\left(\sum_{q=1}^{k}\left|U_{q}\right|^{t}\right)<a^{t} m^{-1} .
$$

But $F \subseteq \bigcup_{i, j, q} \varphi_{i, j}^{-1}\left(U_{q}\right)$; hence $F$ has a covering by sets of diameter less than $\frac{1}{2} \delta$ with the same bound on the $t$ th power of the diameters. Repeating this argument, we see that there are sets $V_{i}$ covering $F$, of diameters at most $2^{-n} \delta$ 
such that $\sum\left|V_{i}\right|^{t}<a^{t} m^{-1}$. It follows that $\mathscr{H}^{t}(F) \leq a^{t} m^{-1}$, and $\operatorname{dim} t<s$, a contradiction.

We now show that (2.1) implies that $\operatorname{dim}_{H} F=\underline{\operatorname{dim}}_{B} F=\overline{\operatorname{dim}}_{B} F$.

Theorem 3. Let $(F, d)$ be a nonempty compact metric space, and suppose that there exist $\lambda_{0}, a>0$ such that for any set $N \subseteq F$ with $|N|<\lambda_{0}$ there is a mapping $\varphi: N \rightarrow F$ such that

$$
\operatorname{ad}(x, y) \leq|N| d(\varphi(x), \varphi(y))
$$

for $x, y \in N$. Then $\operatorname{dim}_{H} F=\underline{\operatorname{dim}}_{B} F=\overline{\operatorname{dim}}_{B} F$.

Proof. We always have $\operatorname{dim}_{H} F \leq \underline{\operatorname{dim}}_{B} F \leq \overline{\operatorname{dim}}_{B} F$. Suppose that $s>$ $\operatorname{dim}_{H} F$, then

$$
0=\mathscr{H}^{s}(F)<a^{s}
$$

Choose an integer $k$ and subsets $U_{1}, \ldots, U_{k}$ of $F$ with $0<\left|U_{i}\right|<\min \{a / 2$, $\left.\lambda_{0}\right\}$ for each $i$, such that $F \subseteq \bigcup_{i=1}^{k} U_{i}$ and

$$
\sum_{i=1}^{k}\left|U_{i}\right|^{s}<a^{s} .
$$

By hypothesis there exist $\varphi_{i}: U_{i} \rightarrow F$ such that

$$
\operatorname{ad}(x, y) \leq\left|U_{i}\right| d\left(\varphi_{i}(x), \varphi_{i}(y)\right)
$$

if $x, y \in U_{i}$.

Let $J_{q}=\left\{i_{1}, \ldots, i_{q}: 1 \leq i_{j} \leq k\right\}$ be the set of $q$ term sequences, and let $J=\bigcup_{q=1}^{\infty} J_{q}$ be the set of all such finite sequences. Let $J_{\infty}=\left\{i_{1}, i_{2}, \ldots: 1 \leq\right.$ $\left.i_{j} \leq k\right\}$ be the corresponding set of infinite sequences. For each $\left(i_{1}, \ldots, i_{q}\right) \in$ $J_{q}$ let

$$
U_{i_{1}, \ldots, i_{q}}=\varphi_{i_{1}}^{-1}\left(\varphi_{i_{2}}^{-1}\left(\cdots\left(\varphi_{i_{q}}^{-1}(F)\right)\right)\right)
$$

(Of course at each stage the image under $\varphi_{i}^{-1}$ is a subset of $U_{i}$, so some of these sets may be empty.) If $x, y \in U_{i_{1}, \ldots, i_{q}}$ repeated use of $(2.5)$ gives

$$
d(x, y) \leq a^{-q}\left|U_{i_{1}}\right| \cdots\left|U_{i_{q}}\right| d\left(\varphi_{i_{1}}\left(\cdots\left(\varphi_{i_{q}}(x)\right)\right)\right), \varphi_{i_{1}}\left(\cdots\left(\varphi_{i_{q}}(y)\right)\right) .
$$

Hence

$$
\left|U_{i_{1}, \ldots, i_{q}}\right| \leq a^{-q}\left|U_{i_{1}}\right| \cdots\left|U_{i_{q}}\right||F| \text {. }
$$

Let $0<b=\min _{1 \leq i \leq k} a^{-1}\left|U_{i}\right|$ and note that $\max _{1 \leq i \leq k} a^{-1}\left|U_{i}\right|<\frac{1}{2}$. Suppose that $\varepsilon<|F|$. For each sequence $\left(i_{1}, i_{2}, \ldots\right) \in J_{\infty}$ curtail the sequence at the least value of $q$ such that

$$
b \varepsilon<\left(a^{-1}\left|U_{i_{1}}\right|\right)\left(a^{-1}\left|U_{i_{2}}\right|\right) \cdots\left(a^{-1}\left|U_{i_{q}}\right|\right)|F| \leq \varepsilon .
$$

Let $S$ be the finite set of finite sequences $\left(i_{1}, \ldots, i_{q}\right)$ obtained in this way. There is exactly one sequence in $S$ that is an initial sequence of each infinite 
sequence in $J$. Repeated use of the inclusion $F \subset \bigcup_{i=1}^{k} U_{i}=\bigcup_{i=1}^{k} \varphi_{i}^{-1}(F)$ gives that

$$
F \subset \bigcup_{i_{1}, \ldots, i_{q} \in S} U_{i_{1}, \ldots, i_{q}} .
$$

Further, (2.7) together with repeated application of $(2.4)$, that $\sum_{i=1}^{k}\left(a^{-1}\left|U_{i}\right|\right)^{s}<$ 1 , gives

$$
(b \varepsilon)^{s} \operatorname{card} S<\sum_{i_{1}, \ldots, i_{q} \in S}\left(a^{-1}\left|U_{i_{1}}\right|\right)^{s} \cdots\left(a^{-1}\left|U_{i_{q}}\right|\right)^{s}|F|^{s}<|F|^{s} .
$$

Hence $F$ may be covered by at most $|F|^{s}(b \varepsilon)^{-s}$ sets of diameter at most $\varepsilon$. It follows that $\varlimsup_{\lim } n(\varepsilon) \varepsilon^{s}<\infty$, so that $\overline{\operatorname{dim}}_{B} F \leq s$, and hence $\overline{\operatorname{dim}}_{B} F \leq$ $\operatorname{dim}_{H} F$.

Note that it would be possible to extend Theorem 3 in the way that Theorem 1 has been extended to Theorem 2 .

Our next result concerns transformations in the opposite direction, from a set onto small neighbourhoods of itself. We give conditions for the Hausdorff and box counting dimensions to be equal, as well as for the critical Hausdorff measure to be finite.

Theorem 4. Let $(F, d)$ be a compact metric space with $\operatorname{dim}_{H} F=s<\infty$. Suppose that there exists $a, r_{0}>0$ such that for any ball $B$ in $F$ of radius $r<r_{0}$ there is a mapping $\psi: F \rightarrow B$ satisfying

$$
\operatorname{ard}(x, y) \leq d(\psi(x), \psi(y))
$$

for $x, y \in F$. Then $\overline{\lim } n(\varepsilon) \varepsilon^{s} \leq a^{-s}$, where $n(\varepsilon)$ is the maximum number of disjoint closed balls of radius $\varepsilon$ with centres in $F$, so that $\underline{\operatorname{dim}}_{B} F=\overline{\operatorname{dim}}_{B} F=s$ and $\mathscr{H}^{s}(F)<\infty$.

Proof. Suppose that for some $\varepsilon<\min \left\{a^{-1}, r_{0}\right\}$ we have

$$
n(\varepsilon) \varepsilon^{s}>a^{-s} \text {. }
$$

Choose $t>s$ such that

$$
n(\varepsilon) \varepsilon^{t}>a^{-t}
$$

Let $B_{1}, \ldots, B_{n(\varepsilon)}$ be closed disjoint balls of radius $\varepsilon$ with centres in $F$ and write $\delta=\min _{i \neq j} d\left(B_{i}, B_{j}\right)>0$. By hypothesis there exist mappings $\psi_{i}: F \rightarrow$ $B_{i}$ for $1 \leq i \leq n(\varepsilon)$ such that

$$
\operatorname{a\varepsilon d}(x, y) \leq d\left(\psi_{i}(x), \psi_{i}(y)\right) .
$$

For any positive integer $q$ and distinct sequences $i_{1}, \ldots, i_{q}$ and $j_{1}, \ldots, j_{q}$, let $m$ be the least integer such that $i_{m} \neq j_{m}$. Applying (2.13) $(m-1)$ times

$$
\begin{gathered}
d\left(\psi_{i_{1}} \circ \cdots \circ \psi_{i_{q}}(F), \psi_{j_{1}} \circ \cdots \circ \psi_{j_{q}}(F)\right) \geq(a \varepsilon)^{m-1} d\left(B_{i_{m}}, B_{j_{m}}\right) \\
\geq(a \varepsilon)^{q} \delta .
\end{gathered}
$$


We may define a measure $\mu$ on the class of finite unions of sets $\psi_{i_{1}} \circ \cdots \circ \psi_{i_{q}}(F)$ by letting $\mu\left(\psi_{i_{1}} \circ \cdots \circ \psi_{i_{q}}(F)\right)=n(\varepsilon)^{-q}$. This extends to a measure $\mu$ on the $\sigma$-algebra generated by these sets, which in turn give rise to an outer measure $\mu$ on the subsets of $F$. Let $U$ be any subset of $F$ with $|U|<a \leq \delta$ and let $q$ be the least integer such that

$$
(a \varepsilon)^{q} \delta>|U| \geq(a \varepsilon)^{q+1} \delta .
$$

Then by (2.14) $U$ intersects at most one set $\psi_{i_{1}} \circ \cdots \circ \psi_{i_{q}}(F)$, so

$$
\begin{aligned}
\mu(U) & \leq n(\varepsilon)^{-q} \\
& \leq(a \varepsilon)^{q t} \\
& \leq(a \varepsilon \delta)^{-t}|U|^{t}
\end{aligned}
$$

by (2.12) and (2.15). Now suppose that $F \subset \bigcup_{i=1}^{\infty} U_{i}$ where $\left|U_{i}\right|<a \varepsilon \delta$ for each $i$. Then $1=\mu(F) \leq \sum_{i=1}^{\infty} \mu\left(U_{i}\right) \leq(a \varepsilon \delta)^{-t} \sum_{i=1}^{\infty}\left|U_{i}\right|^{t}$. Hence $\mathscr{H}^{t}(F)>0$, implying that $\operatorname{dim}_{H} F \geq t>s$, contrary to hypothesis. We conclude that $n(\varepsilon) \varepsilon^{s} \leq a^{-s}$ for all sufficiently small $\varepsilon$, giving $\overline{\operatorname{dim}}_{B} F \leq s$. Finally, given a maximal set of disjoint balls of radii $\varepsilon$ with centres in $F$, the set of concentric balls of radii $2 \varepsilon$ cover $F$. It follows that $\mathscr{C}^{s}(F)<\infty$.

\section{EXAMPLES AND APPLICATIONS}

Example 1. Dynamical repellers. Let $M$ be a Riemann manifold, and $f$ an expanding map of class $C^{1+\eta}$ on $M$ (that is the tangent map of $f$ satisfies a Hölder condition with exponent $\eta>0$ ). We assume that $J$ is a compact set satisfying the following properties:

(i) $f$ is expanding on $J$, that is there are constants $c>0$ and $\alpha>1$ such that

$$
\left\|\left(T_{x} f^{n}\right)(u)\right\| \geq c \alpha^{n}\|u\|
$$

for $x \in J$ and $n \geq 1$, when $T_{x} f$ is the tangent map of $F$ at $x$.

(ii) $J$ is invariant under $f$ and $f^{-1}$, that is $f(J) \subset J$ and $f^{-1}(J) \subset J$.

(iii) $f$ is topologically mixing on $J$, so that for every open set $U$ intersecting $J$ there is an $n>0$ such that $J \subset f^{n}(U)$.

The set $J$ is called a (mixing) repeller for $f$, and often may be identified with the Julia set of $f$.

We use our results to show that if $f$ is conformal, that is if $T_{x} f$ is always a scalar multiple of an isometry, then $J$ has equal box counting and Hausdorff dimensions. Moreover, $J$ has positive finite Hausdorff measure at the critical value, a result obtained by Ruelle [9] and Bowen [2] using Markov partitions and Gibbs measures, who also give the Hausdorff dimension of $J$ in terms of the pressure of certain functions. 
Theorem. Let $f$ be a $C^{1+\eta}$ conformal mapping on a Riemann manifold $M$ with mixing repeller $J$. If $s=\operatorname{dim}_{H} J$, then $\underline{\operatorname{dim}}_{B} J=\overline{\operatorname{dim}}_{B} J=s$ and $0<\mathscr{H}^{s}(J)<\infty$.

Proof. We may assume that the Riemann metric $d$ is adapted to $f$, so that (3.1) becomes

$$
1<\alpha<\left\|T_{w} f\right\| \leq \beta<\infty
$$

for all $w \in J$, the upper bound following from the compactness of $J$. By the compactness of $J$, there exist $r_{0}>0$ and $\gamma>1$ such that for each $w \in J$, if $x, y \in B_{r_{0}}(w)$ then

$$
d(f(x), f(y)) \geq \gamma d(x, y) .
$$

(We write $B_{r_{0}}(w)$ for the closed ball in $M$ of centre $w$ and radius $r_{0}$.) We may further assume, using the mean value theorem on $f$ and its local inverse and the Hölder condition on $T_{w} f$, that $r_{0}$ is chosen so that, for some constant $c<\frac{1}{2} r_{0}^{-\eta}$,

$$
1-c d(x, y)^{\eta} \leq \frac{d(f(x), f(y))}{\left\|T_{w} f\right\| d(x, y)} \leq 1+c d(x, y)^{\eta}
$$

whenever $w \in J$ and $x, y \in B_{r_{0}}(w)$ with $x \neq y$. If $f^{i}(x), f^{i}(y) \in B_{r_{0}}\left(f^{i}(w)\right)$ for $0 \leq i \leq n-1$ then (3.2) gives

$$
2 r_{0} \geq d\left(f^{n-1}(x), f^{n-1}(y)\right) \geq \gamma^{n-1-i} d\left(f^{i}(x), f^{i}(y)\right)
$$

so that

$$
\begin{aligned}
\prod_{i=0}^{n-1}\left(1-c\left(2 r_{0}\right)^{\eta} \gamma^{-i \eta}\right) & \leq \frac{d\left(f^{n}(x), f^{n}(y)\right)}{\left\|T_{w} f\right\|\left\|T_{f(w)} f\right\| \cdots\left\|T_{f^{n-1}(w)} f\right\| d(x, y)} \\
& \leq \prod_{i=0}^{n-1}\left(1+c\left(2 r_{0}\right)^{\eta} \gamma^{-i \eta}\right) .
\end{aligned}
$$

Thus

$$
b_{1} \leq \frac{d\left(f^{n}(x), f^{n}(y)\right)}{\left\|T_{w} f^{n}\right\| d(x, y)} \leq b_{2}
$$

for constants $0<b_{1}<b_{2}<\infty$ for $x \neq y$, provided that $f^{i}(x), f^{i}(y) \in$ $B_{r_{0}}\left(f^{i}(w)\right)$ for $0 \leq i \leq n-1$. Let $w \in J, 0<r<\min \left\{r_{0}, r_{0} b_{2}^{-1}\right\}$. Choose the least non-negative integer $n$ such that

$$
r_{0} / \beta \leq b_{2} r\left\|T_{w} f^{n}\right\|<r_{0} .
$$

If $x, y \in B_{r}(w)$, then using (3.5) for successively larger $n$ gives $f^{i}(x), f^{i}(y) \in$ $B_{r_{0}}\left(f^{i}(w)\right)$ for $0 \leq i \leq n$. Hence

$$
\frac{b_{1} r_{0}}{b_{2} \beta r} d(x, y) \leq d\left(f^{n}(x), f^{n}(y)\right) \leq \frac{r_{0}}{r} d(x, y) .
$$


Taking $\varphi$ as the restriction of $f^{n}$ to $B_{r}(w) \cap J$ in Theorems 1 and 3, it follows that $\mathscr{H}^{s}(J)>0$ and $\operatorname{dim}_{B} J=\overline{\operatorname{dim}}_{B}=s$.

Since $f$ is topologically mixing on the compact set $J$, we may fix a positive integer $m$ such that $f^{m}\left(B_{r_{1}}(w)\right) \supseteq J$ for every $w \in J$, where $r_{1}=b_{1} r_{0} / b_{2} \beta$. There is a number $v$ such that

$$
d\left(f^{m}(x), f^{m}(y)\right) \leq v d(x, y)
$$

for all $x, y \in J$. Let $w \in J$ and $r<r_{1}$. As in (3.6) above there exists $n$ such that $f^{n}: B_{r}(w) \rightarrow B_{r_{0}}\left(f^{n}(w)\right)$ is an injection, satisfying

$$
\frac{r_{1}}{r} d(x, y) \leq d\left(f^{n}(x), f^{n}(y)\right) \leq \frac{r_{0}}{r} d(x, y) \quad\left(x, y \in B_{r}(w)\right) .
$$

By invariance of dimension in the Riemann manifold $M, f^{n}\left(B_{r}(w)\right) \supseteq$ $B_{r_{1}}\left(f^{n}(w)\right)$. Restricting $f^{n}: B_{r}(w) \cap J \rightarrow f^{n}\left(B_{r}(w)\right) \cap J$, let $h: B_{r_{1}}\left(f^{n}(w)\right) \cap$ $J \rightarrow B_{r}(w) \cap J$ be the restriction of the inverse of $f^{n}$ to $B_{r_{1}}\left(f^{n}(w)\right) \cap J$. Thus

$$
d(x, y) \leq \frac{r_{0}}{r} d(h(x), h(y)) \quad\left(x, y \in B_{r_{1}}\left(f^{n}(w) \cap J\right)\right) .
$$

Let $g: J \rightarrow B_{r_{1}}\left(f^{n}(w)\right) \cap J$ be any (set theoretic) right inverse of the surjection $f^{m}: B_{r_{1}}\left(f^{n}(w)\right) \cap J \rightarrow J$. From (3.7)

$$
d(x, y) \leq v d(g(x), g(y)) .
$$

Thus if $\psi=h \circ g: J \rightarrow B_{r}(w) \cap J$, then

$$
d(x, y) \leq \frac{v r_{0}}{r} d(\psi(x), \psi(y)) .
$$

It follows from Theorem 4 that $\mathscr{l}^{s}(J)<\infty$, as required.

Example 2. Self-similar sets. For $i=1, \ldots, k$ let $\psi_{i}: \mathbf{R}^{n} \rightarrow \mathbf{R}^{n}$ be contracting similarity transformations, i.e.

$$
d\left(\psi_{i}(x), \psi_{i}(y)\right)=c_{i} d(x, y)
$$

where $0<c_{i}<1$ and $d$ is the Euclidean metric. It is well known (see Hutchinson [4]) that there exists a unique nonempty compact $F \subset \mathbf{R}^{n}$ that is self-similar in the sense that

$$
F=\bigcup_{i=1}^{k} \psi_{i}(F)
$$

In general $F$ will be a fractal. It is easy to see that if $z \in F$ then there is a (not necessarily unique) infinite sequence $\left(i_{1}, i_{2}, \ldots\right)$ such that the sequence of compact sets $\psi_{i_{1}} \circ \cdots \circ \psi_{i_{q}}(F)$ decreases to $z$ as $q \rightarrow \infty$. Hence given $r<|F|$ there exists a least integer $q$ such that

$$
\operatorname{ar} \leq c_{i_{1}} c_{i_{2}} \cdots c_{i_{q}}|F| \leq r
$$

where $a=\min _{1 \leq i \leq k} c_{i}$. Writing $\psi=\psi_{i_{1}} \circ \cdots \circ \psi_{i_{q}}$

$$
\operatorname{ard}(x, y) /|F| \leq d(\psi(x), \psi(y)) \leq r d(x, y) /|F|
$$


by (3.8). Thus taking $x=\psi^{-1}(z)$ we see that $\psi$ maps $F$ into the ball of centre $z$ and radius $r$. It follows by Theorem 4 that, if $s=\operatorname{dim}_{H} F$, then $\mathscr{H}^{s}(F)<\infty$ and $\operatorname{dim}_{H} F=\underline{\operatorname{dim}}_{B} F=\overline{\operatorname{dim}}_{B} F$.

Suppose now that the $\psi_{1}$ satisfy the open set condition of Hutchinson [4], namely that there exists an open set $U$ such that

$$
U \supset \bigcup_{i=1}^{k} \psi_{i}(U)
$$

with this union disjoint. Let $\lambda<1$ be given. Then for every infinite sequence $\left(i_{1}, i_{2}, \ldots\right)$ with $1 \leq i_{j} \leq k$, there is a least integer $q$ such that $a \lambda \leq c_{i_{1}} c_{i_{2}} \cdots c_{i_{q}} \leq \lambda$. Let $S$ be the set of curtailed sequences $\left(i_{1}, \ldots, i_{q}\right)$ obtained in this way. It follows from iterating (3.10) that

$$
U \supset \bigcup_{s} \psi_{i_{1}} \circ \cdots \circ \psi_{i_{q}}(U)
$$

with this union disjoint. Moreover, it is easy to show that $F \subseteq$ $\bigcup_{s} \psi_{i_{q}} \circ \cdots \circ \psi_{i_{q}}(\bar{U})$. Let $N \subset F$ have $|N|=\lambda$ and let $U_{1}, \ldots, U_{m}$ denote the sets $\psi_{i_{1}} \circ \cdots \circ \psi_{i_{q}}(U)$ with $\left(i_{1}, \ldots, i_{q}\right) \in S$ that have closures which intersect $N$. Then $a \lambda|U| \leq\left|U_{j}\right| \leq \lambda|U|$. The $U_{j}$ are disjoint and are contained in a ball of radius $\lambda(1+|U|)$ with center in $N$. Comparing volumes it follows that $|m| \leq m_{0}=(1+|U|)^{n} a^{-n}$, which is independent of $\lambda$. Let $N_{j}=N \cap \bar{U}_{j}(1 \leq j \leq m)$ and let $\varphi_{j}: N_{j} \rightarrow F$ be the restriction of $\left(\psi_{i_{1}} \circ \cdots \circ \psi_{i_{q}}\right)^{-1}$ to $N_{j}$. Then $d(x, y) \leq|N| d\left(\varphi_{j}(x), \varphi_{j}(y)\right)$ for $x, y \in N_{j}$ and it follows from Theorem 2 that $0<\mathscr{H}^{s}(F)$ where $s=\operatorname{dim}_{H} F$.

Thus if the open set condition holds, then $0<\mathscr{H}^{s}(F)<\infty$ where $s=$ $\operatorname{dim}_{H} F$. A further consequence of the previous paragraph is that any $z \in F$ belongs to at least one and at most $m_{0}$ of the sets $\psi_{i_{1}} \circ \cdots \circ \psi_{i_{q}}(F)$ for each $q$. Since $F=\bigcup \psi_{i_{1}} \circ \cdots \circ \psi_{i_{q}}(F)$, where the union is over the set $J_{q}$ of all sequences $\left(i_{1}, \ldots, i_{q}\right)$ of length $q$, we have, using the scaling property of Hausdorff measures, that

$$
\mathscr{H}^{s}(F) \leq \sum_{J_{q}} \mathscr{H}^{s}\left(\psi_{i_{1}} \circ \cdots \circ \psi_{1_{q}}(F)\right)=\sum_{j_{q}}\left(c_{i_{1}} \cdots c_{i_{1}}\right)^{s} \mathscr{H}^{s}(F) \leq m_{0} \mathscr{H}^{s}(F) .
$$

Thus $1 \leq\left(\sum_{i=1}^{k} c_{i}^{s}\right)^{q} \leq m_{0}$ for each integer $q$, so it follows that $\operatorname{dim}_{H} F$ is the solution of $\sum_{i=1}^{k} c_{i}^{s}=1$

These ideas may be extended further, for example to the situation where the $\psi_{i}$ are conformal contractions of class $C^{1+\eta}$ on a subset of a Riemann manifold. The methods of Example 1 may be used to estimate $d(\psi(x), \psi(y))$ and the invariant sets will have equal Hausdorff and box-counting dimensions. Moreover, if the open set condition applies, then $0<\mathscr{H}^{s}(F)<\infty$ at the critical value of $s$. The dimension is given by $\inf \left\{s: \sum\left\|T_{x}\left(\psi_{i_{1}} \circ \cdots \circ \psi_{i_{q}}\right)\right\|^{s}<\infty\right\}$ where the sum is over all finite sequences $\left(i_{1}, \ldots, i_{q}\right)$. For example, we may take $\psi_{1}, \psi_{2}:[-1,1] \rightarrow[-1,1]$ as $\psi_{1}(x)=-x / \alpha$ and $\psi_{2}(x)=g^{-1}(-x / \alpha)$ 
(positive inverse), where $g(x)=1-1.528 x^{2}+0.105 x^{4}+\cdots$ is the fixed point function of the Feigenbaum renormalization equation and $\alpha=2.503 \ldots$ (see Rand [8] or Falconer [3] \$8.7). It follows that the closed invariant Cantorlike attractor of $g$ has positive finite $s$-dimensional Hausdorff measure, where $s=0.538 \ldots$.

Example 3. Recurrent sets. This is essentially a generalisation of the previous example, which is discussed in Bedford [1]. For $i=1, \ldots, k$ let $\psi_{i}: \mathbf{R}^{n} \rightarrow$ $\mathbf{R}^{n}$ be contracting similarity transformations of ratios $0<c_{i}<1$. Let $T$ be a subset of $\{1,2, \ldots, k\} \times\{1,2, \ldots, k\}$, and assume that $T$ satisfies a transitivity condition that if $1 \leq i, j \leq k$ then there is a sequence $i=$ $i_{1}, i_{2}, \ldots, i_{q}=j$ with $\left(i_{m}, i_{m+1}\right) \in T$ for $1 \leq m \leq q-1$. Let $J_{q}=$ $\left\{\left(1=i_{1}, i_{2}, \ldots, i_{q}\right):\left(i_{m}, i_{m+1}\right) \in T, 1 \leq m \leq q-1\right\}$ and $J=\{(1=$ $\left.\left.i_{1}, i_{2}, \ldots\right):\left(i_{m}, i_{m+1}\right) \in T, 1 \leq m \leq \infty\right\}$. Let $F_{q}$ be the decreasing sequence of sets given by

$$
F_{q}=\bigcup_{J_{q}} \psi_{i_{1}} \circ \cdots \circ \psi_{i_{q}}(E)
$$

where $E$ is some compact set with $\psi_{i}(E) \subseteq E$ for each $i$. We are interested in the dimension and measure of the set

$$
F=\bigcap_{q=1}^{\infty} F_{q},
$$

which is independent of the initial $E$ chosen.

Let $p=\max _{(i, j)} \min \left\{t: i=i_{1}, \ldots, i_{t}=j\right.$ for some sequence $i_{1}, \ldots, i_{t}$ with $t>1\}$, and let $0<a=\min _{i} c_{i}<1$. If $z \in F$ there is a (not necessarily unique) infinite sequence $\left(1=i_{1}, i_{2}, \ldots\right)$ with $\left(i_{m}, i_{m+1}\right) \in T$ for $1 \leq m<\infty$ and with the sequence of compact sets $\psi_{1} \circ \psi_{i_{2}} \circ \cdots \circ \psi_{i_{q}}(F)$ decreasing to $z$. Hence given $r<|F|$ there is a least integer $q$ such that

$$
a r \leq c_{1} c_{i_{2}} \cdots c_{i_{q}}|F| \leq r .
$$

with $\left(i_{m}, i_{m+1}\right) \in T$. There is a sequence $i_{q}, i_{q+1}, \ldots, i_{q+t}=1$ where $1 \leq t \leq$ $p-1$ and $\left(i_{m}, i_{m+1}\right) \in T$ for $q \leq m \leq q+t-1$. Thus

$$
a^{p} r \leq c_{1} c_{i_{2}} \cdots c_{i_{q+1-1}}|F| \leq r .
$$

Let $\psi=\psi_{1} \circ \psi_{i_{2}} \circ \cdots \circ \psi_{i_{q+1-1}}$. Then

$$
a^{p} r d(x, y) /|F| \leq d(\psi(x), \psi(y)) \leq r d(x, y) /|F| .
$$

Taking $x=\psi^{-1}(z)$ we see, since $\left(i_{q+t-1}, 1\right) \in T$, that $\psi: F \rightarrow B_{r}(z) \cap F$. It follows from Theorem 3 that, if $s=\operatorname{dim}_{H} F$, then $\mathscr{H}^{s}(F)<\infty$ and $\operatorname{dim}_{H} F=$ ${\operatorname{dim}_{B}} F=\overline{\operatorname{dim}}_{B} F$.

To show that $\mathscr{H}^{s}(F)>0$ we require the open set condition (3.10) to hold. Let $\lambda<1$. For every sequence $\left(i_{1}, i_{2}, \ldots\right) \in J$ there is a least $q$ such that $a \lambda \leq$ $c_{i_{1}} c_{i_{2}} \cdots c_{i_{q}} \leq \lambda$. Let $S \subseteq \bigcup_{q=1}^{\infty} J_{q}$ be the set of curtailed sequences obtained 
in this way. Let $N \subseteq F$ have $|N|=\lambda$. Just as in the case of self-similar sets, there is an integer $m_{0}$ such that at most $m_{0}$ of the sets $\psi_{i_{1}} \circ \cdots \circ \psi_{i_{q}}(U)$ with $\left(i_{1}, \ldots, i_{q}\right) \in S$ have closures intersecting $N$. We work with each of these sets $U_{0}=\psi_{i_{1}} \circ \cdots \circ \psi_{i_{q}}(U)$ in turn. For some $2 \leq t \leq p$ we can find a sequence $1=j_{1}, \ldots, j_{t}=i_{q}$ with $\left(j_{m}, j_{m+1}\right) \in T$ for $1 \leq m \leq t-1$. If $\psi=\left(\psi_{j_{1}} \circ \cdots \circ \psi_{j_{t}}\right) \circ\left(\psi_{i_{\mathrm{i}}} \circ \cdots \circ \psi_{i_{1}}\right)^{-1}$ then

$$
d(x, y) \leq \lambda a^{-p} d(\psi(x), \psi(y)) .
$$

Moreover, $\psi: U_{0} \cap N \rightarrow F$. Hence by Theorem 2, $0<\mathscr{H}^{s}(F)$.

We can now find $\operatorname{dim}_{H} F$. For convenience, assume that the sets $\psi_{i}(F)$ $(1 \leq i \leq k)$ are disjoint, this assumption may be weakened to the open set condition by proceeding along the lines of Example 2. For each $i \quad(1 \leq i \leq k)$ let

$$
F^{(i)}=\bigcap_{q=1}^{\infty}\left\{\bigcup \psi_{i} \circ \psi_{i_{2}} \circ \cdots \circ \psi_{i_{q}}(E):\left(i, i_{2}\right),\left(i_{m}, i_{m+1}\right) \in T\right\} .
$$

As above $0<\mathscr{H}^{s}\left(F^{(i)}\right)<\infty$ for each $i$; by the transitivity of $T, s=\operatorname{dim}_{H} F^{(i)}$ is independent of $i$. By assumption

$$
F^{(j)}=\bigcup\left\{\psi_{j}\left(F^{(i)}\right):(j, i) \in T\right\}
$$

is a disjoint union, so that

$$
\left(\begin{array}{c}
\mathscr{H}^{s}\left(F^{(1)}\right) \\
\vdots \\
\mathscr{H}^{s}\left(F^{(k)}\right)
\end{array}\right)=M C^{s}\left(\begin{array}{c}
\mathscr{H}^{s}\left(F^{(1)}\right) \\
\vdots \\
\mathscr{H}^{s}\left(F^{(k)}\right)
\end{array}\right)
$$

where $M$ denotes the matrix with $(i, j)$ th entry of 1 if $(i, j) \in T$ and 0 otherwise, and

$$
C^{s}=\left(\begin{array}{ccc}
c_{1}^{s} & & 0 \\
& \ddots & \\
0 & & c_{k}^{s}
\end{array}\right) .
$$

Since $M C^{s}$ is an irreducible matrix with non-negative entries, the PerronFrobenius theory tells us that

$$
\left(\begin{array}{c}
\mathscr{C}^{s}\left(F^{(1)}\right) \\
\vdots \\
\mathscr{H}^{s}\left(F^{(k)}\right)
\end{array}\right)
$$

is, to within a scalar, the unique eigenvector of $M C^{s}$ with positive entries, with corresponding eigenvalue the unique eigenvalue of largest absolute value, equal to the spectral radius of $M C^{S}$. This eigenvalue is strictly decreasing with $s$, so that $\operatorname{dim}_{H} F^{(i)}$ is the value of $s$ making the largest eigenvalue of $M C^{s}$ equal to 1 . 


\section{REFERENCES}

1. T. Bedford, Dimension and dynamics for fractal recurrent sets, J. London Math. Soc. (2) 33 (1986), 89-100.

2. R. Bowen, Hausdorff dimension of quasi-circles, Pub. Math. I.H.E.S. 50 (1979), 11-25.

3. K. Falconer, The geometry of fractal sets, Cambridge Univ. Press, Cambridge, England, 1985.

4. J. E. Hutchinson, Fractals and self-similarity, Indiana Univ. Math. J. 30 (1981), 713-747.

5. B. B. Mandelbrot, The fractal geometry of nature, W. H. Freeman, San Francisco, Ca., 1982.

6. J. McLaughlin, A note on Hausdorff measures of quasi-self-similar sets, Proc. Amer. Math. Soc. 100 (1987), 183-186.

7. C. Tricot, Two definitions of fractional dimension, Math. Proc. Cambridge Philos. Soc. 91 (1982), 57-74.

8. D. Rand, University and renormalisation in dynamical systems in New directions in dynamical systems, London Math. Soc. Lecture Notes 127 (1988), 1-56.

9. D. Ruelle, Bowen's formula for the Hausdorf dimension of selfsimilar sets, in Scaling and self-similarity in physics-renormalization in statistical mechanics and dynamics, Progr. Phys. 7 (1983).

School of Mathematics, University Walk, Bristol BS8 1TW, ENGLAND 